\title{
On the Existence of Simple Reducible Vector Bundles on Complex Surfaces of Algebraic Dimension Zero
}

By

\author{
Matei ToMA*
}

Simple holomorphic vector bundles on compact complex manifolds admit coarse moduli spaces which are only locally Hausdorff in general, cf. [N], (for another approach in the more general setting of simple coherent sheaves on compact complex spaces see [K-O]). A question which arises is to decide when these moduli spaces are not empty, hence the question of existence of simple vector bundles.

On the other hand one has a notion of irreducible vector bundles (cf. [E-F], [B-L]). These are holomorphic vector bundles $E$ which admit no coherent subsheaf $F$ with $0<\operatorname{rank} F<\operatorname{rank} E$. In contrast with the algebraic case there exist such bundles on some nonalgebraic manifolds by $[\mathrm{E}-\mathrm{F}]$ and $[\mathrm{B}-\mathrm{L}]$. Even vector bundles of stronger irreducibility type are shown to exist ([T]). Irreducible vector bundles are always simple, while reducible ones have many endomorphisms in general.

The aim of this paper is to determine the range of Chern classes $c_{1}, c_{2}$ of reducible simple rank 2 vector bundles on minimal complex surfaces of algebraic dimension zero, extending the results for surfaces without divisors of [B-F2]. Apart from some precise exceptions this range will be the same as that of reducible rank 2 vector bundles determined in [B-L] for nonalgebraic surfaces; see the theorem for the exact statement. Here we illustrate the result by the

Corollary. Let $X$ be a minimal surface with $a(X)=0$. Then there exists $a$ simple reducible bundle of rank 2 on $X$ with $c_{1}=0$ and given $c_{2} \in \mathbb{Z}$ if and only if $c_{2} \geqslant 0$, excepting exactly the following cases:

Communicated by K. Saito, December 21, 1989.

1991 Mathematics Subject Classification: 32J15, 32L05.

* Department of Mathematics, INCREST, Bd. Pacii 220, 79622 Bucharust, Romania. 
$-X$ is a torus and $c_{2}=0$,

$-X$ is in class VII and $c_{2}=0$, unless $b_{2}(X)=0, X$ without divisors and $c_{1}(X) \in$ $2 \mathrm{NS}(X)$

- X is a $\mathrm{K} 3$ surface with curves and $c_{2}=0,1,2,3$.

$-X$ is a K3 surface without curves and $0 \leqslant c_{2}<\inf \left\{-\xi^{2} \mid 0 \neq \xi \in \mathrm{NS}(X)\right\}$.

Determining the above range is equivalent to determining which topological rank 2 vector bundles admit simple reducible holomorphic structures, since by a classical result of $\mathrm{Wu}$ topological vector bundles on surfaces are characterized up to isomorphisms by their rank and Chern classes $c_{1}, c_{2}$.

I wish to thank Constantin Bănică for the useful discussions concerning this paper.

\section{§1. Preliminaries and Statement of the Main Result}

We denote by $X$ a compact complex connected nonsingular surface. If $X$ is minimal and its algebraic dimension $a(X)=0$, we have from the classification of surfaces (cf. [B-P-V]) the following three situations:

1) $X$ is a complex torus

2) $X$ is in class VII i.e. $\operatorname{kod}(X)=-\infty$ and $b_{1}(X)=1$

3) $X$ is a $\mathrm{K} 3$ surface i.e. $K_{X}=\mathcal{O}_{X}$ and $q(X)=0$ where $K=K_{X}$ is the canonical bundle.

When $a(X)=0, X$ has at most $h^{1,1}(X)+2$ irreducible curves ([B-P-V]). If moreover $X$ is a complex torus it has none at all.

Let now $E$ denote a holomorphic rank 2 vector bundle on $X$, henceforth called simply "bundle". $E$ is by definition simple if $\operatorname{End}(E) \cong C . \quad E$ is called reducible or filtrable if it admits a coherent subsheaf of rank 1. Equivalently, $E$ is filtrable if $H^{0}(E \otimes L) \neq 0$ for some $L$ in $\operatorname{Pic}(X)$. It is worth noting that the condition $(C)$ in $[I]$ says that the cotangent bundle is filtrable. One can prove that $E$ is filtrable if and only if it admits a devissage i.e. an exact sequence

$$
0 \rightarrow L_{1} \stackrel{\alpha}{\rightarrow} E \stackrel{\beta}{\rightarrow} L_{2} \otimes \mathscr{I}_{Y} \rightarrow 0
$$

where $L_{1}, L_{2} \in \operatorname{Pic}(X)$ and $Y$ is a 2-codimensional analytic subspaces of $X$ or empty (cf. [E-F]).

Conversely, one uses extensions of type (1) with $L_{1}, L_{2} \in \operatorname{Pic}(X), Y$ a 2codimensional subspace of $X$ in order to show the existence of reducible holomorphic structures on a given topological rank 2 vector bundle. These ex- 
tensions are parametrized by $\operatorname{Ext}^{1}\left(L_{2} \otimes \mathscr{I}_{Y}, L_{1}\right)$. By Serre (cf. [O-S-S] Ch. I, $\S 5$ ), the middle term of an extension (1) is locally free if and only if the image of the corresponding element $\theta \in \operatorname{Ext}^{1}\left(L_{2} \otimes \mathscr{I}_{Y}, L_{1}\right)$ through the canonical mapping

$$
\operatorname{Ext}^{1}\left(\mathscr{I}_{Y} \otimes L_{2}, L_{1}\right) \rightarrow H^{0}\left(X, \mathcal{E} x t^{1}\left(\mathscr{I}_{Y} \otimes L_{2}, L_{1}\right)\right)
$$

generates the sheaf $\mathcal{E} x t^{1}\left(L_{2} \otimes \mathscr{I}_{Y}, L_{1}\right)$.

Let $L=L_{2}^{V} \otimes L_{1}$. Since in our case $\operatorname{Ham}\left(L_{2} \otimes \mathscr{I}_{Y}, L_{1}\right) \cong L_{2}^{V} \otimes L_{1}=L$ the exact sequence of the first terms of the Ext spectral sequence becomes:

$$
0 \rightarrow H^{1}(X, L) \rightarrow \operatorname{Ext}^{1}\left(\mathscr{I}_{Y}, L\right) \rightarrow H^{0}\left(X, \mathcal{E x t}^{1}\left(\mathscr{I}_{Y}, L\right)\right) \rightarrow H^{2}(X, L) .
$$

For $Y$ as above we set $l(Y)=h^{0}\left(\mathcal{O}_{Y}\right)=$ length $_{C}\left(\mathcal{O}_{Y}\right)$.

\section{Remarks.}

1) Serre's condition is fulfilled in the following cases (cf. [B-L]):

i) $Y$ is a locally complete intersection (hence $\mathcal{E}_{x} t^{1}\left(\mathscr{I}_{Y}, L\right) \cong \mathcal{O}_{Y}$ ) and $H^{2}(X, L)=0$.

ii) $Y$ is a locally complete intersection, $L^{V} \otimes K \cong \mathcal{O}(D)$ with $D$ an effective divisor, and $Y \subset D$ as analytic spaces. (By duality we get that the map $H^{0}\left(X, \mathcal{E x t}^{1}\left(\mathcal{I}_{Y}, L\right)\right) \rightarrow H^{2}(X, L)$ is zero $)$.

iii) $Y$ consists of simple points, $l(Y)>1, H^{2}(X, L) \neq 0, L^{V} \otimes K=\mathcal{O}(D)$ and $Y \cap \operatorname{supp} D=\varnothing$. (One can find sections in $H^{0}\left(X, \mathcal{E} x t^{1}\left(\mathscr{I}_{Y}, L\right)\right) \cong H^{0}\left(Y, \mathcal{O}_{Y}\right)$ mapped to zero in $H^{2}(X, L)=H^{0}(X, O(D))^{V}$ having nonzero components in each fiber $\mathcal{O}_{Y, y}, y \in Y$ ).

2) Serre's condition is not fulfilled if $l(Y)=1$ (hence $Y$ is a simple point), $L^{V} \otimes K=\mathcal{O}(D)$ and $Y$ is not on supp $D$.

To see this note that the morphism $H^{0}\left(X, \mathcal{E x t}^{1}\left(\mathscr{I}_{Y}, L\right)\right) \rightarrow H^{2}(X, L)$ is the dual of the restriction

$$
H^{0}\left(X, L^{V} \otimes K\right) \rightarrow H^{0}\left(Y, L^{V} \otimes K_{\mid Y}\right)
$$

Irreducible bundles are obviously simple. For a reducible bundle given by (1) we have the

\section{Lemma 1.}

i) If $E$ is simple then $H^{0}\left(L_{2}^{V} \otimes L_{1}\right)=0$.

ii) If $H^{0}\left(L_{2}^{V} \otimes L_{1}\right)=H^{0}\left(L_{1}^{V} \otimes L_{2} \otimes \mathscr{I}_{Y}\right)=0$ and (1) doesn't split then $E$ is simple.

In particular if $X$ has no divisors $E$ is simple if and only if $L_{1} \not \models L_{2}$ and (1) doesn't split. 
Proof. i) For a nonzero section $\varphi$ in $H^{0}\left(L_{2}^{V} \otimes L_{1}\right)$ the composition

$$
E \rightarrow L_{2} \otimes \mathscr{I}_{Y} \hookrightarrow L_{2} \stackrel{\varphi}{\rightarrow} L_{1} \rightarrow E
$$

would give a nonconstant endomorphism of $E$.

ii) It is enough to show that there are no nonzero noninvertible elements in $\operatorname{End}(E)$.

Assume now $\varepsilon \in \operatorname{End}(E)$ is such an element. Since $\beta \circ \varepsilon \circ \alpha=0$ we have a commutative diagram

$$
\begin{aligned}
0 & \rightarrow L_{1} \stackrel{\alpha}{\rightarrow} E \stackrel{\beta}{\rightarrow} \mathbb{L}_{2} \otimes \mathscr{I}_{Y} \rightarrow 0 \\
r \downarrow & \downarrow \varepsilon \quad \downarrow \delta \\
0 & \rightarrow L_{1} \stackrel{\alpha}{\rightarrow} E \stackrel{\beta}{\rightarrow} L_{2} \otimes \mathscr{L}_{Y} \rightarrow 0
\end{aligned}
$$

where $\gamma$ and $\delta$ are homotheties or zero. Using the Ker-Coker Lemma one finds that $\gamma$ and $\delta$ cannot be simultaneously isomorphisms nor simultaneously zero.

If $\delta=0, \gamma \neq 0$ there exists $\psi: E \rightarrow L_{1}$ such that $\alpha \circ \psi=\varepsilon$. Hence $\alpha \circ \gamma=\varepsilon \circ \alpha=$ $\alpha \circ \psi \circ \alpha$ which gives $r=\psi \circ \alpha$ and (1) splits.

In the same way we exclude the case $r=0, \delta \neq 0$.

We make the following notations: For $c_{1} \in \mathrm{NS}(X)$ and $c_{2} \in H^{4}(X, \mathbb{Z}) \cong \mathbb{Z}$

$$
\begin{aligned}
& \Delta=\Delta\left(c_{1}, c_{2}\right)=\frac{1}{2}\left(c_{2}-\frac{1}{4} c_{1}^{2}\right) \\
& M\left(c_{1}\right)=\left\{-\frac{1}{2}\left(\frac{c_{1}}{2}-\xi\right)^{2}: \xi \in N S(X)\right\} \\
& m\left(c_{1}\right)=\inf M\left(c_{1}\right) \\
& m^{\prime}\left(c_{1}\right)=\left\{\begin{array}{lll}
\inf \left(M\left(c_{1}\right) \backslash\left\{m\left(c_{1}\right)\right\}\right) & \text { if } & M\left(c_{1}\right) \neq\left\{m\left(c_{1}\right)\right\} \\
\infty & \text { if } & M\left(c_{1}\right)=\left\{m\left(c_{1}\right)\right\}
\end{array}\right.
\end{aligned}
$$

Notice that $m\left(c_{1}\right) \geqslant 0$ by Kodaira's theorem (hence $m^{\prime}\left(c_{1}\right)>0$ ). For a bundle $E$ one has $2\left(\Delta(E)-m\left(c_{1}(E)\right)\right) \in \mathbb{Z}$. One can immediately see that $m\left(c_{1}\right)=0$ for $c_{1} \in 2 \mathrm{NS}(X)$ and $m^{\prime}(0)=\infty$ when $\mathrm{NS}(X)=0$. (For more examples see Remark 4) at the end of the paragraph).

In [B-L] Bănică and Le Potier gave necessary and suficient conditions for the existence of filtrable holomorphic structures on a topological vector bundle on a nonalgebraic surface in terms of its Chern classes. In our particular case this gives the following result:

There exists a filtrable bundle on $X$ having Chern classes $c_{1} \in \mathrm{NS}(X), c_{2} \in \mathbb{Z}$ if and only if $\Delta \geqslant m\left(c_{1}\right)$, excepting the precise case when $X$ is $\mathbb{K} 3, m\left(c_{1}\right)=0$ and 
$\Delta=1 / 2$.

We can now state our main result.

Theorem. If $X$ is a minimal surface with $a(X)=0$ there exists a simple reducible rank 2 vector bundle on $X$ having Chern classes $c_{1} \in \mathrm{NS}(X), c_{2} \in \mathbb{Z}$, if and only if

$$
\Delta\left(c_{1}, c_{2}\right) \geqslant m\left(c_{1}\right)
$$

excepting exactly the following cases:

1) if $X$ is a torus

$$
\Delta\left(c_{1}, c_{2}\right)=m\left(c_{1}\right)=0
$$

2) if $X$ is in class VII

$$
\Delta\left(c_{1}, c_{2}\right)=m\left(c_{1}\right)=0,
$$

unless $b_{2}(X)=0, X$ without divisors and $c_{1} \in c_{1}(X)+2 \mathrm{NS}(X)$

3) if $X$ is a K3 surface

$$
\begin{aligned}
& m\left(c_{1}\right)=0 \text { and } 0 \leqslant \Delta\left(c_{1}, c_{2}\right)<\sup \left\{m^{\prime}\left(c_{1}\right), 2\right\} \\
& \Delta\left(c_{1}, c_{2}\right)=m\left(c_{1}\right)=\frac{1}{4} \\
& \Delta\left(c_{1}, c_{2}\right)=m\left(c_{1}\right)=\frac{1}{2} .
\end{aligned}
$$

The proof will be given in the following paragraphs. In doing this we may restrict ourselves to surfaces admitting divisors the other cases having been dealt with in [B-F2]. In particular we do not consider here the case of tori. We only note that for tori $X$ with $a(X)=0$ the intersection form on $\operatorname{NS}(X)$ is negative definite (cf. [B-F1]). Hence $m\left(c_{1}\right)=0$ if and only if $c_{1} \in 2 \mathrm{NS}(X)$. Thus the only topological rank 2 vector bundles admitting reducible but not simple reducible holomorphic structures are the twistings of the trivial rank 2 bundle by elements in $\mathrm{NS}(X)$.

We now make some remarks about the statement:

3) In the class VII there exist surfaces $X$ without curves and with $b_{2}(X)=0$ (Inoue surfaces for example, cf. [I]), which make the exception in 2) worth noting. Since $b_{2}(X)=0$, for every such surface $X$ there exists an unramified covering $X^{\prime}$ such that $c_{1}\left(X^{\prime}\right)=0$, thus illustrating the corresponding exception of the Corollary. 
4) For a $\mathrm{K} 3$ surface $X, H^{1}(X, \mathcal{O})=0$ hence $\operatorname{Pic}(X) \cong \mathrm{NS}(X) \hookrightarrow H^{2}(X, \mathscr{Z})$. $H^{2}(X, Z)$ is free and the intersection form is even. Moreover for $L$ in $\operatorname{Pic}(X)$, $L^{2}=-2$ implies $L=\mathcal{O}( \pm C)$ for some effective divisor $C$. On the other hand if $C$ is an irreducible curve then it is rational and $C^{2}=-2$. For these facts we refer to $[\mathrm{B}-\mathrm{P}-\mathrm{V}]$. We recall also that in our case $(a(X)=0)$ the intersection form is negative definite (cf. $[\mathrm{B}-\mathrm{L}] \S 2.6$ ).

We derive some consequences.

If $m\left(c_{1}\right)=\frac{1}{4}$ then $X$ admits divisors.

When $m\left(c_{1}\right)=0$ it follows $c_{1} \in 2 \mathrm{NS}(X)$ and $m^{\prime}\left(c_{1}\right)<2$ if and only if there are curves on $X$ (in this case $m^{\prime}\left(c_{1}\right)=1$ ).

When $X$ is a special $\mathrm{K} 3$ surface of type $g$ (i.e. NS $(X)$ is cyclic generated by $L$ with $\left.L^{2}=2 g-2, \mathrm{cf} .[L]\right)$ and $g<0$, one gets

$$
\sup \left\{m^{\prime}(0), 2\right\}=m^{\prime}(0)=1-g
$$

and $m\left(c_{1}(L)\right)=(1-g) / 4$.

One can get an even wider domain of exceptions when $\mathrm{NS}(X)=0$. Since in this case $m^{\prime}(0)=\infty$, there are no simple reducible bundles (this can also be seen directly).

5) Comparing our result with the range of Chern classes given by the existence theorem for irreducible bundles of $[\mathrm{B}-\mathrm{L}], \S 5$, we find that there exist topological vector bundles admitting simple holomorphic structures and reducible holomorphic structures but not simple reducible holomorphic structures. This happens for instance, on special $\mathbb{K} 3$-surfaces of type $g$ with $g<-1$, for $c_{1}=0$ and $2 \leqslant \Delta<1-g$. It never happens if $X$ is a torus with $a(X)=0$ (use also [B-L] Proposition 4.7.).

6) On the other hand there exist topological vector bundles admitting simple reducible holomorphic structures but not irreducible structures, on tori and $\mathrm{K} 3$ surfaces of zero algebraic dimension.

This can be seen using Proposition 4.3. and 4.7. in [B-L] and our theorem. The simplest examples one gets are on tori for $c_{1}=0, c_{2}=1$ and on $\mathbb{K} 3$ surfaces with curves for $c_{1}=$ the class of a $(-2)$-curve and $c_{2}=1$ or 2 .

Before the proof of the theorem we shall make the following remark.

The tensor product of a devissage of $E$ with a line bundle $L$ gives a devissage of $E \otimes L=E^{\prime}$ and one has 


$$
\begin{aligned}
& \Delta\left(E^{\prime}\right)=\Delta(E) \\
& c_{1}\left(E^{\prime}\right)=c_{1}(E)+2 c_{1}(L) \\
& m\left(c_{1}\left(E^{\prime}\right)\right)=m\left(c_{1}(E)\right) \\
& m^{\prime}\left(c_{1}\left(E^{\prime}\right)\right)=m^{\prime}\left(c_{1}(E)\right)
\end{aligned}
$$

so we have to consider only the classes $c_{1}+2 \mathrm{NS}(X)$ of $c_{1}$ modulo $2 \mathrm{NS}(X)$. In particular it will be enough for our problem to consider devissages with $L_{2}$ trivial:

$$
0 \rightarrow L \rightarrow E \rightarrow \mathscr{I}_{Y} \rightarrow 0
$$

More precisely a topological rank 2-vector bundle characterized by $\left(c_{1}, \Delta\right)$ admits simple reducible holomorphic structures if and only if there exists some simple holomorphic bundle given by an extension of type (2) having the same discriminant $\Delta$, and first Chern class congruent modulo $2 \mathrm{NS}(X)$ with $c_{1}$.

From (2) one derives

$$
\begin{aligned}
& c_{1}(E)=c_{1}(L) \\
& c_{2}(E)=l(Y) .
\end{aligned}
$$

Finally note that if $E$ is given by (2) and $\Delta(E)=m\left(c_{1}(E)\right)$, then $Y=\varnothing$ and $L^{2}=-8 m\left(c_{1}\right) . \quad$ Indeed $\Delta(E)=\frac{1}{2}\left(l(Y)-\frac{1}{4} L^{2}\right) \geqslant-\frac{1}{8} L^{2} \geqslant m\left(c_{1}(E)\right)$.

\section{§2. Proof of the Case: $X$ in Class VII}

Let now $X$ be in class VII, $c_{1} \in \mathrm{NS}(X), c_{2} \in \boldsymbol{Z}$. Let $\Delta=\Delta\left(c_{1}, c_{2}\right)$.

a) If $\Delta>m\left(c_{1}\right)$ choose $L_{1}$ in $\operatorname{Pic}(X)$ such that $c_{1}\left(L_{1}\right) \in c_{1}+2 \mathrm{NS}(X)$ and $m\left(c_{1}\right)=-\frac{1}{2}\left(\frac{1}{2} c_{1}\left(L_{1}\right)\right)^{2}$. Twist $L_{1}$ by $L_{0}$ in $\operatorname{Pic}_{0}(X)$ in order to have for $L=L_{1} \otimes L_{0}$ :

$$
H^{0}\left(L^{V} \otimes K\right)=H^{0}(L)=H^{0}\left(L^{V}\right)=0 .
$$

This is possible since $\operatorname{Pic}_{0}(X) \cong \boldsymbol{C} \backslash\{0\}$ and the elements in $\operatorname{Pic}(X)$ admitting nontrivial sections form a countable subset. As we have said, we can assume $c_{1}=c_{1}(L)$. The assumption $\Delta>m\left(c_{1}\right)$ implies $c_{2}>0$.

The condition $H^{0}\left(L^{V} \otimes K\right)=0$ ensures the existence of an extension (2) with $E$ locally free having the wanted Chern classes if $Y$ is the union of $c_{2}$ distinct simple points. The other two vanishings ensure the simplicity of $E$ by Lemma 1. 
b) If $\Delta=m\left(c_{1}\right)>0$ take $\mathbb{L}$ as before, and $Y=\varnothing$. One has $\Delta=-\frac{1}{8} c_{1}(\mathbb{L})^{2}=$ $m\left(c_{1}\right)$. Then, by Riemann-Roch's formula

$$
\begin{aligned}
& h^{1}(\mathbb{L})=4 \Delta+\frac{1}{2} \mathbb{L} \cdot \mathbb{K} \\
& h^{1}\left(\mathbb{L}^{V}\right)=4 \Delta-\frac{1}{2} \mathbb{L} \cdot \mathbb{K}+h^{0}(\mathbb{L} \otimes \mathbb{K})
\end{aligned}
$$

and at least one of these numbers is positive.

Consider a corresponding nontrivial extension

$$
\begin{aligned}
& 0 \rightarrow \mathbb{L} \rightarrow E \rightarrow \mathcal{O} \rightarrow 0 \quad \text { or } \\
& 0 \rightarrow \mathbb{L}^{V} \rightarrow E \rightarrow \mathcal{O} \rightarrow 0
\end{aligned}
$$

which will give a bundle $\mathbb{E}$ of the wanted type.

c) Let now $\Delta=m\left(c_{1}\right)=0$.

First we shall show that if $E$ is a simple reducible vector bundle of rank 2 having these Chern classes, then necessarily $b_{2}(X)=0, X$ has no divisors and $c_{1} \in c_{1}(X)+2 N S(X)$. We can assume that $\mathbb{E}$ has a devissage of type (2). The hypothesis implies $Y=\varnothing$ and $c_{1}(L)^{2}=0$. Thus $\mathbb{E}$ is the middle term of a nontrivial extension

$$
0 \rightarrow \mathbb{L} \rightarrow \mathbb{E} \rightarrow \mathcal{O} \rightarrow 0
$$

with $L^{2}=0, h^{0}(L)=0$ (as $\mathbb{E}$ is simple) and $h^{0}\left(\mathbb{L}^{V} \otimes \mathbb{K}\right)=1$. For this last fact, first deduce $L \circ K=0$ from $L^{2}=0$ (examine $(K+n L)^{2}, n \in \mathbb{Z}$ ), then apply Riemann-Roch for $L$.

Thus $K=L\left(\sum_{i=1}^{k} r_{i} C_{i}\right)$ with $r_{i}$ nonnegative integers and $C_{i}$ irreducible curves on $X$. If $K \cdot \mathbb{C}_{i}<0$ then $\mathbb{C}_{i}^{2}<0$ (examine again $\left.\left(K+n C_{i}\right)^{2}\right)$ hence $\mathbb{C}_{i}$ is exceptional which is absurd. It follows that $K^{2}=K \cdot L+\sum_{i=1}^{k} r_{i} K \cdot C_{i} \geqslant 0$. But for our surfaces $b_{2}(X)=-K^{2}$, hence $b_{2}(X)=0$.

If $X$ has no curves, one has $K=\mathbb{L}\left(h^{0}\left(K^{V} \otimes L\right)=1 !\right)$, hence $c_{1}=c_{1}(X)$ and the statement is proved.

Assume now $X$ admits divisors. We'll show that this leads to a contradiction.

Since $a(X)=0, b_{1}(X)=1, b_{2}(X)=0, X$ must be a $\mathbb{H}$ opf surface (i.e. its universal covering manifold is $\left.\mathbb{C}^{2} \backslash\{0\}\right)$ by theorem 34 in $\mathbb{K}$ odaira's paper $[\mathbb{K}]$. Hence one has the following form for the canonical bundle: 


$$
K=\left\{\begin{array}{l}
\mathcal{O}\left(-C_{1}-C_{2}\right), \text { if } X \text { admits two irreducible curves } C_{1}, C_{2}, \\
\mathcal{O}(-(m+1) C), \text { if } X \text { admits only one irreducible curve } C
\end{array}\right.
$$

where $m$ is a positive integer depending on the transformation group on $C^{2} \backslash\{0\}$ giving $X$. Denote $D_{1}=\sum_{i=1}^{k} r_{i} C_{i} \geqslant 0$, so that $L=K\left(-D_{1}\right)$. There are divisors $D_{2}>0, D_{3}>0$ such that $K=\mathcal{O}\left(-D_{2}-D_{3}\right)$. We have a commutative diagram

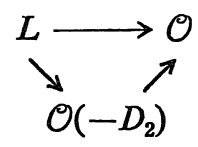

where all the arrows are natural inclusions. Passing to cohomology in dimension one, since $H^{1}\left(\mathcal{O}\left(-D_{2}\right)\right)=0$ by Riemann-Roch (recall $b_{2}(X)=0$ ), one finds that the natural map

$$
H^{1}(L) \rightarrow H^{1}(\mathcal{O})
$$

is zero.

Since (3) is nontrivial the connecting homomorphism in

$$
0 \rightarrow H^{0}(L) \rightarrow H^{0}(E) \rightarrow H^{0}(\mathcal{O}) \stackrel{\delta}{\rightarrow} H^{1}(L) \rightarrow H^{1}(E)
$$

is nontrivial and $H^{0}(E)=H^{0}(L)=0$. Twisting (3) by $L^{V}$ one finds

$$
\begin{aligned}
& 0 \rightarrow L \rightarrow E \rightarrow \mathcal{O} \rightarrow 0 \\
& 0 \rightarrow \mathcal{O} \rightarrow E^{V} \rightarrow L^{V} \rightarrow 0
\end{aligned}
$$

with vertical arrows given by functoriality by the natural inclusion

$$
\begin{aligned}
\mathcal{O} \hookrightarrow L^{V}=\mathcal{O}\left(D_{1}+D_{2}+D_{3}\right) . \text { Hence } & \\
& H^{0}(\mathcal{O}) \stackrel{\delta}{\rightleftharpoons} H^{1}(L) \\
& \downarrow \downarrow \\
& \downarrow \\
0 \rightarrow H^{0}(\mathcal{O}) \rightarrow H^{0}\left(E^{V}\right) \rightarrow & H^{0}\left(L^{V}\right) \rightarrow H^{1}(\mathcal{O})
\end{aligned}
$$

is commutative. (Counting dimensions shows that $\delta$ and the first vertical map are isomorphisms). Since the second vertical map is zero we obtain

$$
h^{0}\left(E^{V}\right)=h^{0}(\mathcal{O})+h^{0}\left(L^{V}\right)=2
$$

Any element in $H^{0}\left(E^{V}\right)$ induces twisting (3) by $E^{V}$ a commutative diagram 


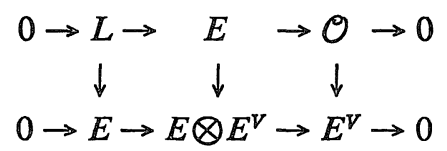

Hence

$$
\begin{aligned}
H^{0}(\mathcal{O}) & \stackrel{\delta}{\rightarrow} H^{1}(L) \\
\downarrow & \downarrow \\
0 \rightarrow H^{0}\left(E \otimes E^{V}\right) \rightarrow H^{0}\left(E^{V}\right) & \rightarrow H^{1}(E)
\end{aligned}
$$

is commutative. We want to prove that the arrow $H^{0}\left(E^{V}\right) \rightarrow H^{1}(E)$ in (5) is zero thus finding $h^{0}\left(E \otimes E^{V}\right)=2$, a contradiction.

Let $\alpha_{1}, \alpha_{2}$ linearly independent in $H^{0}\left(E^{V}\right)$ with $\alpha_{1}$ induced by the morphism $L \rightarrow E$ of (3). By (4) the morphism $H^{1}(L) \stackrel{H^{1}\left(\alpha_{1}\right)}{\longrightarrow} H^{1}(E)$ is zero. Denote by $\beta_{1}$ the morphism $E \rightarrow \mathcal{O}$ appearing in (3). Then $\beta_{1} \circ \alpha_{2} \neq 0$ hence $\beta_{1} \circ \alpha_{2}$ must be proportional to the natural inclusion $L \rightarrow \mathcal{O}$. We denoted also by $\alpha_{2}$ the morphism $L \rightarrow E$ inducing $\alpha_{2} \in H^{0}\left(E^{V}\right)$. Thus $H^{1}\left(\beta_{1} \circ \alpha_{2}\right)=0$ as we have already seen. Since by (4), $H^{1}\left(\beta_{1}\right)$ is injective we find that $H^{1}(L) \stackrel{H^{1}\left(\alpha_{2}\right)}{\longrightarrow} H^{1}(E)$ is zero.

As $\alpha_{1}, \alpha_{2}$ generate $H^{0}\left(E^{V}\right)$, it follows from (5) that the arrow $H^{0}\left(E^{V}\right) \rightarrow H^{1}(E)$ must be null and $E$ is not be simple: a contradiction.

d) When $\Delta=m\left(c_{1}\right)=0, X$ in class VII without divisors, $b_{2}(X)=0$ and $c_{1} \in c_{1}(X)$ $+2 N S(X)$ one derives the existence of simple reducible holomorphic structures out of a non-trivial extension

$$
0 \rightarrow K \rightarrow E \rightarrow \mathcal{O} \rightarrow 0
$$

(use $h^{1}(K)=1$ and Lemma 1 ).

\section{§3. Proof of the Case: $X$ a K3 Surface}

For $X$ a $\mathrm{K} 3$ surface with $a(X)=0$ we shall use the properties already mentioned in Remark 4) of paragraph 1 . We also use the fact that in this case $(a(X)=0)$ an isomorphism $\mathcal{O}\left(D_{1}\right) \cong \mathcal{O}\left(D_{2}\right)$ implies $D_{1}=D_{2}$ for two divisors $D_{1}$, $D_{2}$.

Let $c_{1} \in N S(X), c_{2} \in \mathbb{Z}$ and $\Delta=\Delta\left(c_{1}, c_{2}\right)$. One sees that $4 m\left(c_{1}\right), 4 \Delta$ and $2\left(\Delta-m\left(c_{1}\right)\right)$ are integers. We shall subdivide the proof into cases depending on the values of $m\left(c_{1}\right)$ and $\Delta$. These cases cover the interesting range in the following way: 


\begin{tabular}{|c|c|c|c|c|}
\hline$\Delta-m\left(c_{1}\right)$ & 0 & $\frac{1}{4}$ & $\frac{1}{2}$ & $>\frac{1}{2}$ \\
\hline 0 & $c$ & $c$ & $c$ & $c$ \\
\hline$\frac{1}{2}$ & $e$ & $d$ & $b$ & $b$ \\
\hline 1 and $\frac{3}{2}$ & $f$ and $g$ & $a$ & $a$ or $b$ & $a$ or $b$ \\
\hline$\geqslant 2$ & $h$ & $a$ & $a$ or $b$ & $a$ or $b$ \\
\hline
\end{tabular}

\section{a) $m\left(c_{1}\right)>0, \Delta \geqslant m\left(c_{1}\right)+1$.}

We can choose $L \in \operatorname{Pic}(X)$ such that $c_{1}(L) \in c_{1}+2 \mathrm{NS}(X)$ and $L^{2}=-8 m\left(c_{1}\right)$. Then $L$ is not trivial hence at least one of $H^{0}(L), H^{0}\left(L^{V}\right)$ must be zero. Assume $H^{0}(L)=0$, otherwise replace $L$ by $L^{V}$. Take $Y$ a set of $2\left(\Delta-m\left(c_{1}\right)\right)$ simple points on $X$. If $H^{0}\left(L^{V}\right) \neq 0$ one has $L^{V}=\mathcal{O}(D)$ for some divisor $D>0$ and we can assume the points of $Y$ do not belong to supp $D$. When $H^{0}\left(L^{V}\right)=0$ we have no more restrictions on the choice of $Y$.

In both cases an extension of type (2) produces a locally free sheaf $E$ by Remark 1 in paragraph 1 (note that $H^{2}(L) \cong H^{0}\left(L^{V}\right)$ by duality).

Moreover $E$ has the wanted Chern classes and it is simple by Lemma 1 .

b) $\Delta>m\left(c_{1}\right)>\frac{1}{4}$

We choose $L$ in $\operatorname{Pic}(X)$ such that $c_{1}(L) \in c_{1}+2 \mathrm{NS}(X)$ and $L^{2}=-8 m\left(c_{1}\right)$. We shall show that this choice can be made such that $H^{0}(L)=H^{0}\left(L^{V}\right)=0$; this is clearly true when $L$ is not divisorial. Then taking $Y$ the union of $2\left(\Delta-m\left(c_{1}\right)\right)$ simple points on $X$, the extension (2) produces a simple locally free sheaf $E$ with the requested Chern classes by Remark 1 i) and Lemma 1.

The existence of an element $L$ in $\operatorname{Pic}(X)$ with the properties we need is a consequence of the following facts:

Lemma 2. Let $R$ be a reduced irreducible root system in the $\boldsymbol{R}$-vector space $V$, of type $A, D$ or $E$, let $B=\left(\alpha_{i}\right)_{i}$ be a basis for $R$ and $Q(R)$ the subgroup of $V$ generated by the vectors in $R$.

Then for any $x \in Q(R)$ there is some $y \in x+2 Q(R)$ such that if $y=\sum_{i} y_{i} \alpha_{i}$ with $y_{i} \in \mathbb{Z}$ the following conditions are satisfied:

i) $\langle y, y\rangle \leqslant\langle x, x\rangle$ 
ii) $y$ is a root or one of its coordinates $y_{\dot{8}}$ is zero.

Corollary If $D$ is a divisor on $X$ with $D^{2}=-8 m(D)<-2$ then there exist some divisor $C$ on $X$ with $C \in D+2 N S(X), C^{2}=D^{2}$ and $H^{0}(\mathcal{O}(C))=H^{0}(\mathcal{O}(-C)$ ) $=0$ (i.e. $C$ is neither positive nor negative).

Proof of the Corollary. One can assume supp $D$ connected and $D$ positive otherwise it's easy. For example if $D=D_{1}+D_{2}$ with $D_{1} \cdot D_{2}=0, D_{1}>0, D_{2}>0$ we can choose $C=D-2 D_{2}=D_{1}-D_{2}$.

Since the intersection form on $\operatorname{Div}(X)$ is negative definite supp $D$ is an A-D-E curve (see $[B-P-V]$ p. 74 for this notion). Let $R_{D}$ be the root system of the type given by supp $D$, the roots corresponding to the irreducible components of $D$ forming a basis $B$, with the opposite of the intersection form as scalar product.

Now Lemma 2 produces some divisor $D^{\prime} \in D+2 N S(X)$ with $D^{2} \geqslant D^{2}$, such that $D^{\prime}$ is a root or supp $D^{\prime}$ is strictly contained in supp $D$. The equality $D^{2}=-8 m(D)$ and the definition of $m(D)$ imply $D^{\prime 2}=D^{2}$. It follows that $D^{\prime}$ is not a root (in the A-D-E case all roots have the same length), hence supp $D^{\prime}$ is strictly contained in supp $D$.

If supp $D^{\prime}$ is not connected we are over. If it is, one repeats the same argument starting with $D^{\prime}$ and so on. Finally we get a divisor with a nonconnected support. (Otherwise we get a divisor of the form $k C$ with $C a(-2)$ curve which implies $m(D)=0$ or $\frac{1}{4}$ ).

Proof of Lemma 2. First we can assume that $x$ is minimal in the following sense: for every $x^{\prime} \in x+2 Q(R)\left\langle x^{\prime}, x^{\prime}\right\rangle \geqslant\langle x, x\rangle$.

Let $x=\sum x_{i} \alpha_{i}$. Assume also that all $x_{i}$ are nonzero and $x$ is not a root, otherwise one can take $y=x$.

In particular supp $x$ is connected (supp $x$ has the meaning which can be adapted to divisors as above) and $x$ or $-x$ is positive (i.e. all its coordinates are positive). Indeed, if $x=x_{+}-x_{-}$, where $x_{+}$(resp $x_{-}$) are its positive (resp. negative) part, then

$$
\left\langle x_{+}+x_{-}, x_{+}+x_{-}\right\rangle=\left\langle x_{+}-x_{-}, x_{+}-x_{-}\right\rangle+4\left\langle x_{+}, x_{-}\right\rangle
$$

But $\left\langle x_{+}, x_{-}\right\rangle \leqslant 0$ and we have even equality by minimality of $x$. Then $x_{+}$or $x_{-}$must be null by connectedness.

We shall use the following modulo 2 reduction:

$(\mathbb{R})$ : replace the coordinates of $x$ by 0 or 1 according to their parity. 
If $R$ is of type $A_{n}$ the reduction $(R)$ solves our problem. Indeed, in this case

$$
\langle x, x\rangle=x_{1}^{2}+\left(x_{1}-x_{2}\right)^{2}+\cdots+\left(x_{n-1}-x_{n}\right)^{2}+x_{n}^{2}
$$

and one immediately sees that by $(R)$ we obtain at most $\frac{1}{2}\langle x, x\rangle$ connected components, each such component being a root.

Let now $R$ be of type $D_{n}$ :

$$
\begin{aligned}
& \bigcirc-\alpha_{2} \cdots \alpha_{n-2} \\
& \alpha_{1} \alpha_{n}
\end{aligned}
$$

If $x_{n-2}$ is odd or one of $x_{n-1}, x_{n}$ are even one applies $(R)$ and reasons as above.

If $x_{n-2}$ is even and $x_{n-1}, x_{n}$ are odd apply the following reduction $\left(R^{\prime}\right)$ : replace all odd $x_{i}$ by 1 and all even ones by zero excepting $x_{n-2}$ and all chains of even coordinates connected to $x_{n-2}$ which are replaced by 2 . For example

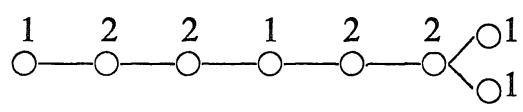

is reduced in this way to

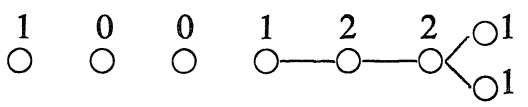

Looking at the quadratic form $\langle x, x\rangle$ one sees that $\left(R^{\prime}\right)$ solves the problem in this case since it doesn't increase $\langle x, x\rangle$ and all the connected components obtained will be roots.

The $E_{6}$-case:

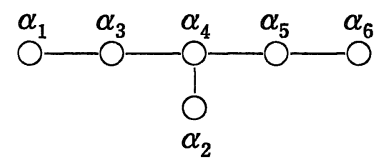

Since after $(R)$ one obtains at most 3 connected components the only difficult case is $\langle x, x\rangle=4$. Noting that

$$
\langle x, x\rangle=\left(\frac{1}{2} x_{1}+x_{2}-x_{3}\right)^{2}+\left(-\frac{1}{2} x_{1}+x_{2}+x_{3}-x_{4}\right)^{2}
$$




$$
\begin{aligned}
& +\left(-\frac{1}{2} x_{1}+x_{4}-x_{5}\right)^{2}+\left(-\frac{1}{2} x_{1}+x_{5}-x_{6}\right)^{2} \\
& +\left(-\frac{1}{2} x_{1}+x_{6}\right)^{2}+\frac{3}{4} x_{1}^{2}
\end{aligned}
$$

one can verify that $(R)$ solves the problem in this case too. We omit the computations. In order to make them shorter one can also assume that

$$
S_{\dot{s}} \in\left[2 x_{\dot{z}}-1,2 x_{\dot{z}}+2\right]
$$

where $S_{\dot{\xi}}$ is the sum of the neighbouring coordinates of $x_{\dot{\xi}}$ on the Dynkin diagram of $R$, otherwise one can contradict the minimality replacing $x_{\dot{i}}$ by some $x_{i}^{\prime}$ of the same parity and leaving all the other coordinates unchanged.

The $E_{7}$-case:

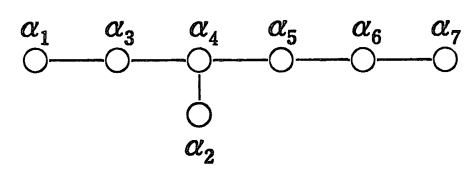

This time the difficult cases are $\langle x, x\rangle \in\{4,6\}$.

$$
\begin{aligned}
\langle x, x\rangle & =\left(\frac{1}{2} x_{1}+x_{2}-x_{3}\right)^{2}+\left(-\frac{1}{2} x_{1}+x_{2}+x_{3}-x_{4}\right)^{2} \\
& +\left(-\frac{1}{2} x_{1}+x_{4}-x_{5}\right)^{2}+\left(-\frac{1}{2} x_{1}+x_{5}-x_{6}\right)^{2} \\
& +\left(-\frac{1}{2} x_{1}+x_{6}-x_{7}\right)^{2}+\left(-\frac{1}{2} x_{1}+x_{7}\right)^{2}+\frac{1}{2} x_{1}^{2}
\end{aligned}
$$

If $x_{6}=x_{7}$ one gets the same quadratic form as for $E_{6}$ and in this case we know the lemma is true. Similarly one can reduce the cases $x_{5}=x_{6}$ and $x_{5}=x_{4}$. The remaining situations can be solved by $(R)$ or $\left(R^{\prime}\right)$ relative to $x_{4}$ instead of $x_{n-2}$.

The $E_{8}$-case:

As before we can restrict ourselves to the cases

$\langle x, x\rangle \in\{4,6\}$ and $x_{8} \neq x_{7} \neq x_{6} \neq x_{5} \neq x_{4}$. This time beside the situations solved by $(R)$ or $\left(R^{\prime}\right)$ we obtain the following solutions

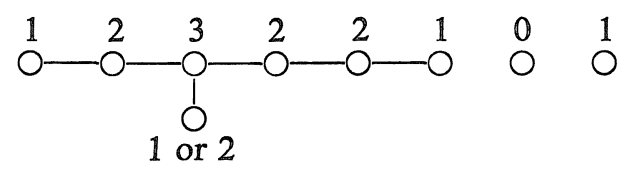

c) $\Delta=m\left(c_{2}\right)$ 
In this case any extension (2) giving a bundle $E$ with the corresponding Chern classes, must have $Y=\varnothing$ and $L^{2}=-8 m\left(c_{1}\right)$.

When $m\left(c_{1}\right)>\frac{1}{2}$ we can choose as in case b) an element $L \in \operatorname{Pic}(X)$ such that $L^{2}=-8 m\left(c_{1}\right), H^{0}(L)=H^{0}\left(L^{V}\right)=0$ and $c_{1}(L) \in c_{1}+2 \mathrm{NS}(X)$.

Hence $L^{2} \leqslant-6$ and by Riemann-Roch's formula

$h^{1}(L) \geqslant-2-\frac{1}{2} L^{2} \geqslant 1$. Thus there exist nontrivial extensions

$$
0 \rightarrow L \rightarrow E \rightarrow \Theta \rightarrow 0
$$

Moreover the middle term $E$ is simple by Lemma 1 .

When $m\left(c_{1}\right) \leqslant \frac{1}{2}$ we show that all extensions (3) with $L^{2}=-8 m\left(c_{1}\right)$ give a non simple bundle E. Indeed, from Riemann-Roch's formula for $\mathcal{E}$ d( $E)$

$$
\chi(X, \operatorname{End}(E))=4 \chi\left(X, \mathcal{O}_{X}\right)-8 \Delta(E)
$$

we get

$2 h^{0}(\operatorname{End}(E)) \geqslant 8-4=4$, and $E$ cannot be simple.

d) $m\left(c_{1}\right)=\frac{1}{4}, \Delta=\frac{3}{4}$

First we state:

\section{Lemma 3. If}

$$
0 \rightarrow L \rightarrow E \rightarrow \mathscr{I}_{Y} \rightarrow 0
$$

is an extension with $L=\mathcal{O}(-D), D$ an effective divisor, $Y$ a simple point belonging to supp $D$ and $E$ locally free, then:

i) $Y \in \operatorname{Reg} D \Rightarrow E$ is simple

ii) $D^{2}=-8$ and $D \in 2 \mathrm{NS}(X) \Rightarrow E$ is not simple.

Before the proof we'll show the existence of simple reducible bundles with corresponding Chern classes.

Choose $L \in c_{1}+2 \mathrm{NS}(X)$ such that $L^{2}=-2$.

If some irreducible component of $D$ appears with multiplicity 1 in $D$ then Reg $D \neq \varnothing$. Taking $Y$ a simple point on Reg $D$ and using Remark 1 and Lemma 3, one obtains the simple reducible bundles we're after out of extensions of the type

$$
0 \rightarrow \mathcal{O}(-D) \rightarrow E \rightarrow \mathscr{I}_{Y} \rightarrow 0 \text {. }
$$

Since $D$ can be seen as a root in the $A, D, E$ root system corresponding to 
supp $D$ one easily sees that $D$ admits an irreducible component of multiplicity 1 in the cases $A_{n}, D_{n}, E_{6}, E_{7}, n \geqslant 1$ (just look at the highest roots !; [H] p. 66). This is also true in the $E_{8}$-case unless $D$ corresponds to the highest root:

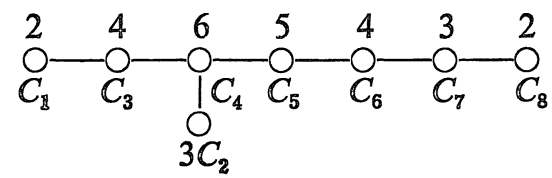

(it is the only root having both $C_{1}$ and $C_{8}$ of multiplicity 2). Here $C_{i}$ denote the irreducible components of $D$.

In this case ( $D$ the highest root in $E_{8}$ ) we consider $D^{\prime}=C_{2}-C_{5}-C_{7} \in D+$ $2 N S(X) . \quad D^{\prime 2}=-6$ and by Lemma 1 , a nontrivial extension

$$
0 \rightarrow \mathcal{O}\left(D^{\prime}\right) \rightarrow E \rightarrow \mathcal{O} \rightarrow 0
$$

provides a simple reducible holomorphic bundle $E$ admitting corresponding Chern classes.

Proof of Lemma 3. i) The long cohomology sequence of

$$
0 \rightarrow \mathscr{I}_{Y} \rightarrow \mathcal{O} \rightarrow \mathcal{O}_{Y} \rightarrow 0
$$

gives $h^{1}\left(\mathscr{I}_{Y}\right)=0, h^{2}\left(\mathscr{I}_{Y}\right)=1$.

Using this in the long cohomology sequence of

$$
0 \rightarrow L \rightarrow E \rightarrow \mathscr{I}_{Y} \rightarrow 0
$$

one finds $h^{0}(E)=0, h^{1}(E)=h^{1}(L), h^{2}(E)=2$.

Passing to sections in

$$
0 \rightarrow E \rightarrow E \otimes E^{V} \rightarrow E^{V} \otimes \mathscr{I}_{Y} \rightarrow 0
$$

we obtain

$$
h^{0}\left(E \otimes E^{V}\right) \leqslant h^{0}\left(E^{V} \otimes \mathscr{I}_{Y}\right) .
$$

We want to show that the subspace of $H^{0}\left(E^{V}\right)$ of sections vanishing on $Y$ is onedimensional (in fact we'll show that this subspace is the image of $i$ in the exact sequence (6) below).

Let $Y=\{p\}$. From

$$
0 \rightarrow \mathcal{O} \rightarrow E^{V} \rightarrow I_{Y}(D) \rightarrow 0
$$

one derives the exact sequences 


$$
\begin{aligned}
& 0 \rightarrow H^{0}(\mathcal{O}) \stackrel{i}{\rightarrow} H^{0}\left(E^{V}\right) \rightarrow H^{0}\left(\mathscr{I}_{Y}(D)\right) \rightarrow 0 \\
& 0 \rightarrow \mathcal{O}_{p} \stackrel{t}{ } \stackrel{\left(-z_{1}, z_{2}\right)}{\longrightarrow} \mathcal{O}_{p}^{2} \stackrel{\left(z_{2}, z_{1}\right)}{\longrightarrow} \mathscr{I}_{Y, p} \rightarrow 0
\end{aligned}
$$

for suitable local coordinates $z_{1}, z_{2}$.

A nonzero section $\alpha$ in $H^{0}\left(\mathscr{I}_{Y}(D)\right)$ can be seen as a section in $H^{0}(\mathcal{O}(D))$ since we have natural inclusions $\mathcal{O} \hookrightarrow \mathscr{I}_{Y}(D) \hookrightarrow \mathcal{O}(D)$. Thus $\alpha$ vanishes of order exactly one on $\operatorname{Reg}(D)$, hence a lifting of it to $H^{0}\left(E^{V}\right)$ cannot vanish in $p$, otherwise from (7) we'd get that $\alpha$ vanishes twice in $p \in \operatorname{Reg}(D)$.

ii) $D>0, D^{2}=-8, D \in 2 \mathrm{NS}(X)$ imply there exists some divisor $C$ such that $D=2 C$. Then taking sections in

$$
0 \rightarrow \mathcal{O}(-C) \rightarrow E(C) \rightarrow \mathscr{I}_{Y}(C) \rightarrow 0
$$

one finds that $h^{0}(E(C))=1$.

A nonzero morphism $\mathcal{O}(-C) \rightarrow E$ induces a devissage

$$
0 \rightarrow \mathcal{O}\left(-C+C^{\prime}\right) \rightarrow E \rightarrow \mathscr{I}_{Z}\left(-C^{\prime}-C\right) \rightarrow 0
$$

with $C^{\prime}$ effective and Lemma 1 tells us that $E$ is not simple.

e) $m\left(c_{1}\right)=0, \Delta=\frac{1}{2}$.

In this case not even holomorphic bundles can be found with these Chern classes by the quoted result of [B.L] (cf. $§ 1$ ).

f) $m\left(c_{1}\right)=0, \Delta=1$.

$$
m\left(c_{1}\right)=0 \text { implies } \quad c_{1} \in 2 \mathrm{NS}(X) .
$$

Then $m^{\prime}\left(c_{1}\right)$ is an integer $\geqslant 1$ with equality exactly when $X$ admits divisors. Two kinds of extensions (2) can appear: either $L=\mathcal{O}$ and $l(Y)=2$ or $L^{2}=-8$ and $l(Y)=0$. As usual $L$ is taken in $2 \mathrm{NS}(X)$. Lemma 1 and an argument parallel to case ii) of Lemma 3 show that both cases give only nonsimple bundles.

g) $m\left(c_{1}\right)=0, \Delta=\frac{3}{2}$.

As before two kinds of extensions can appear: either with $L=\mathcal{O}$ and $l(Y)=3$ or with $L^{2}=-8$ and $l(Y)=1$. The first gives only nonsimple bundles by Lemma 1. For the second we remark that $L \cong \mathcal{O}( \pm 2 C)$ with $C$ effective hence we must choose the situation $L=\mathcal{O}(-2 C)$, otherwise $E$ is not simple by Lemma 1 . Then by Remark $2, Y$ must be a simple point lying on $C$ and case 
ii) of Lemma 3 shows that also in this case $E$ is not simple.

li) $m\left(c_{1}\right)=0, \Delta \geqslant 2$.

When $X$ admits divisors one can choose an irreducible curve $C, Y$ a union of $2(\Delta-1)$ simple points on $X \backslash C$, the extensions

$$
0 \rightarrow \mathcal{O}(-2 C) \rightarrow \mathbb{E} \rightarrow \mathscr{I}_{Y} \rightarrow 0
$$

give simple holomorphic bundles $E$ with the expected Chern numbers by Remark 1 and Lemma 1.

When $X$ admits no divisors one can show as before that there are no simple bundles $E$ with $c_{1}(E) \in 2 \mathrm{NS}(X)$ and $\Delta(E)<m^{\prime}(0)$ (in (2) we should have $L=\mathcal{O}$ ). When $\Delta(E) \geqslant m^{\prime}(0)$ we can choose a holomorphic line bundle $L$ in $2 \mathrm{NS}(X)$, with $L^{2}=-8 m^{\prime}(0), Y$ a union of $2\left(\Delta-m^{\prime}(0)\right)$ simple points and extensions

$$
0 \rightarrow L \rightarrow E \rightarrow \mathscr{I}_{Y} \rightarrow 0
$$

give simple holomorphic bundles admitting the expected Chern classes by Remark 1 and Lemma 1.

The theorem is proved.

\section{References}

[B-L] Bănică C. and Le Potier J., Sur l'existence des fibrés vectorieis holomorphes sur les surfaces non-algébriques, J. reine angew. Math., 378 (1987), 1-31.

[B-P-V] Barth W., Peters C. and Van de Ven A., Compact complex surfaces, Berlin-HeidelbergNew York, (1984).

[B-F1] Brînzănescu V. and Flondor P., Holomorphic 2-vector bundles on nonalgebraic 2tor1, J. reine angen Math., 363 (1985), 47-58.

[B-F2] , Simple filtrable 2-vector bundles on complex surfaces without divisors, Rev. Roumaine Math. Pures Appl., 31 (1986), 507-512.

[E-F] Elencwajg G. and Forster O., Vector bundles on manifolds without divisors and a theorem on deformations, Ann. Inst. Fourier, 32.4 (1982), 25-51.

$[\mathrm{H}]$ Humphreys J.E., Introduction to Lie algebras and representa.ion theo:y, BerlinHeidelberg-New York, (1972).

[I] Inoue M., On surfaces of Class $\mathrm{VII}_{0}$, Inventiones Math., 24 (1974), 269-310.

[K] Kodaira K., On the structure of compact complex analytic surfaces II, Am. J. of Math., 88 (1966), 682-721.

[K-O] Kosarew S. and Okonek C., Global moduli spaces and simple holomorphic burdles, Publ. RIMS, 25 (1989), 1-20.

[L] Le Potier J., Simple connexité des surfaces K3, Astér isque, 126 (1985), 79-90.

[N] Norton V.A., Analytic Moduli of Complex Vector Bundles, Indiana Univ. Math. J., 28.3 (1979), 365-387.

[O-S-S] Okonek C., Schneider M. and Spindler H., Vector bundles on complex projective spaces, Boston-Basel-Stuttgart, (1980).

[T] Toma M., A class of nonalgutraic threefolds, Ann. Insi. Fourier, 39.1 (1989), 239250. 